Research Paper

\title{
Study on the Expressions of PHD and HIF in Placentas from Normal Pregnant Women and Patients with Preeclampsia
}

\author{
Wei Liu1, Shu-Jun Wang², Qi-De Lin ${ }^{1 凶}$ \\ 1. Department of Obstetrics and Gynecology, Ren Ji Hospital, School of Medicine, Shanghai Jiao Tong University, Shanghai, 200127, China; \\ 2. Shanghai Institute of Immunology, Shanghai, 200025, China.
}

$\square$ Corresponding author: Department of Obstetrics and Gynecology, Ren Ji Hospital, School of Medicine, Shanghai Jiao Tong University, Shanghai, 200127, China. E-mail: shlinqide@hotmail.com Tel: +86-0-13916362152 Fax: +86-021-68383802.

(c) Ivyspring International Publisher. This is an open-access article distributed under the terms of the Creative Commons License (http://creativecommons.org/ licenses/by-nc-nd/3.0/). Reproduction is permitted for personal, noncommercial use, provided that the article is in whole, unmodified, and properly cited.

Received: 2013.04.01; Accepted: 2013.11.27; Published: 2014.02.21

\begin{abstract}
Objective: To investigate the relationship between oxygen sensitivity of trophoblast and hypoxia in preeclamptic placenta by the study on the expressions of hypoxia-inducible factor prolyl 4-hydroxylase (PHD) and hypoxia-inducible factor (HIF) in placentas from normal pregnant women and patients with pre-eclampsia.

Methods: Subjects were chosen from the in-patients or the out-patients from May 2003 to May 2004. They were divided into 5 groups: early pregnancy group (EP), 13 cases; middle pregnancy group (MP), 9 cases; late pregnancy group (LP, or control group), 12 cases; preeclampsia (PE) group, 20 cases; gestational hypertension group $(\mathrm{GH}), 10$ cases. The mRNA expressions of PHD-I and -2 and -3 in placentas from all the subjects were assessed by in situ hybridization and Real-time PCR. The expressions of HIF-I $\alpha$ and $-2 \alpha$ in placentas from different groups were assessed by immunohistochemistry and western blot.

Results: PHD-1,-2 and -3 mRNA were mainly expressed in cytoplasm of trophoblast, especially strongly expressed in extravillous trophoblast. During the progress of pregnancy, the expression of PHD-I increased significantly $(R=0.616, P<0.00 I)$. The PHD-ImRNA expression in placentas from $P E$ group decreased significantly compared with that from control group, $P<0.05$. A significant direct correlation between the PHD-I mRNA expression in placentas from PE group and their placenta weight was found $(R=0.457, P<0.05)$. The HIF- $2 \alpha$, not the HIF- $I \alpha$ expression, from $\mathrm{PE}$ group was significantly higher than that from control group, $\mathrm{P}<0.01$; The HIF- $2 \alpha$ expression in trophoblast from $P E$ was inversely correlated to the date of the onset of the disease $(R=-0.730$, $\mathrm{P}<0.0 \mathrm{I})$.

Conclusions: PHD-I played an important role in hypoxic response pathway of trophoblast through modulating the level of HIF- $2 \alpha$. The overly activated hypoxic response pathway of trophoblast in preeclamptic placenta, which is manifested as the result of HIF-2 $\alpha$ over-expression, is the key point to hypoxic dysfunction of trophoblast.
\end{abstract}

Key words: Preeclampsia; Hypoxia-inducible factor; hypoxia-inducible factor prolyl 4-hydroxylase; Trophoblast.

\section{Introduction}

Preeclampsia is characterized by the onset of hypertension and proteinuria during gestation [1]. Advanced-stage clinical symptoms include seizures, renal failure, IUGR, and/or HELLP (hemolysis, elevated liver enzymes, and low platelets) syndrome [2]. If without proper treatment, it can develop into ec- 
lampsia, which was the life-threatening occurrence seizure during pregnancy $[1,2]$. Preeclampsia affects approximately $6-8 \%$ of all pregnancies worldwide [3], with onset of symptoms in the late second or third trimester, most commonly after the 32nd week. In China, hypertensive disorders in pregnancy companied with preeclampsia ranks the second leading cause of maternal mortality [4].

The foremost hypothesis regarding the initiating event in preeclampsia is that insufficient trophoblast invasion and abnormal spiral artery remodeling placental ischemia, which initiates the hypoxia stress and endothelial dysfunction [5]. Those subsequently lead to the clinical symptoms for preeclampsia [6]. Proper cellular response to changes in oxygen tension during normal development or pathological processes is ultimately regulated by the transcription factor hypoxia-inducible factor (HIF) [7]. The active form of HIF is a heterodimeric comprising of subunit $a$, which is oxygen-sensitive and rapidly degraded and inactivated during normoxia, and a constitutively stable subunit $\beta$ [7]. HIF is highly expressed in the low oxygen environment of the placenta in early gestation, playing an important role in placental development and function [8]. HIF has been shown to be abnormal up-regulated in preeclamptic placentas [9-14].

The stability of the HIF-a subunit is regulated by oxygen-dependent prolyl 4-hydroxylation catalyzed by HIF prolyl 4-hydroxylases (PHD) [15]. There are three PHD isoforms that have been identified: PHD-1, -2 and -3 [16, 17]. Under conditions of normoxia, PHD catalyzes the formation of the 4-hydroxylation of HIF targeting it for poly-ubiquitination and proteosomal degradation mediated by the von Hippel-Lindau protein [18]. However, under hypoxic conditions, PHD activity is inhibited, thereby allowing HIF to accumulate and translocate to the nucleus, where it promotes the expression of its target hypoxia-responsive genes [18]. Although PHD is the essential regulator of HIF, and HIF plays a critical role in the pathogenesis of preeclampsia, indicating that PHDs also involves in the preeclampsia. Indeed, a previous work showed that PHD-2 was related to early- preeclamptic placentae but not latepreeclamptic placentae.

In the present work, we comprehensively investigated the expression of PHD and HIF of trophoblast in preeclampsia placenta. Our results showed that preeclampsia was coupled with low level of PHD-1 and high expression of HIF-2 $\alpha$, suggesting that activated hypoxic response is related to hypoxic dysfunction of trophoblast. These results also highlight that hypoxia response will be potential therapy targeted for preeclampsia.

\section{Material and Methods}

\section{Ethics Statement}

This study was conducted according to the principles expressed in the Declaration of Helsinki. The study was approved by Institutional Review Board of Ren Ji Hospital of School of Medicine, Shanghai Jiao Tong University. All patients provided written informed consent for the collection of samples and subsequent analysis.

\section{Subjects Group}

The research subjects investigated in the present work were selected from those who gave birth or accepted treatment in our hospital from May 2003 to May 2004. They were divided in five groups: (1) early pregnancy group: 13 cases, gestational age $<12$ weeks, accepting abortion surgery, normal embryonic development revealed by ultrasonic, deny abnormal reproductive history and history of chronic diseases associated with chronic hypertension, kidney disease and diabetes; (2) middle pregnancy group: 9 cases, gestational age $\geq 12$ weeks and $<28$ weeks, accepting hyponome induced abortion; (3) late pregnant group (control group): 13 cases, gestational age $\geq 28$ weeks $<42$ weeks, accepting elective cesarean, with normal pregnancy and well developed fetal, cesarean was due to breech and social factors, deny previous abnormal reproductive history and history of chronic diseases associated with chronic hypertention, kidney disease and diabetes, this group was the control group to both the preeclampsia group and the gestational hypertension group; (4) preeclampsia group: 20 cases, accepting hospitalization and sectional cesarean delivery, preeclampsia, diagnostic criteria, deny history of chronic diseases associated with chronic hypertention, kidney disease and diabetes; (5) gestational hypertension group: 11 cases, with pregnancy-induced hypertension treatment and cesarean delivery, deny a history of chronic hypertension, kidney disease, diabetes and other chronic diseases. We got approve from all of the research objects for this research. The clinical parameters of control groups and preeclampsia groups were provided in the Table 1.

\section{Sampling}

Under aseptic conditions, a few pieces of placenta tissue, after being taken out from middle pregnant and late pregnant group, with the size about 0.5 $\times 0.5 \times 0.5 \mathrm{~cm}^{3}$ was taken from center parts of maternal side by sterile scissors and tweezers. For early pregnancy group, the whole placental villus was taken after abortion surgery. All of these tissues were rinsed with sterile saline 2 or 3 times to wash away the hemorrhage followed by placing in $10 \%$ neutral buff- 
ered formalin or freezing in liquid nitrogen for further analysis.

\section{In situ hybridization}

In situ hybridization for detecting PDH-1, -2 and -3 were performed with BIO-AP REMBRANDT® Universal DISH \& Detection Kit following manufacture's instruction. For each gene's detection, three specific probes were used. Primer sequences for in situ hybridization were listed in Supplementary Material: Table S1. The Images of staining were taken using Axioplan 2 imaging system (Zeiss, Germany).

\section{Real-time PCR}

Total RNA was prepared using Guanidinium Thiocyanate-Phenol-Chloroform Extraction. cDNA was obtained by using High Capacity cDNA Reverse Transcription Kit (Life technology). Real-time PCR was performed with SYBR green probe in an ABI Prism 7900 according to the manufacturer's specified parameters. The data of Real-time PCR was analyzed by SDS 2.0 (Applied Biosystems, USA) GAPDH was used as control. Primer sequences for RT-PCR are listed in Supplementary Material: Table S2.

\section{Immunohistochemistry}

Formalin-fixed paraffin embedded placenta tissue were treated with standard processing including slicing, baked pieces, dewaxing, dehydration, blocking and closing. The immunohistochemical staining was performed with All-in-One Kit for Immunohistochemical Staining for Tissues (Invitrogene, USA) following manufacture's protocol. Anti-HIF-1 $\alpha$ (Abcam, ab85886) and anti-HIF-2 $\alpha$ (Abcam, ab199) for staining were used with a dilution of $1 / 1000$ and $1 / 500$, respectively.

\section{Western blot}

$50 \mathrm{mg}$ placental tissue or chorionic villi, placed in $0.5 \mathrm{ml}$ pre-cooling of the cell lysate, were used to make protein suspension with a high-speed homogenizer. The supernatant after high-speed centrifugation $\left(12000 \times \mathrm{g}, 4^{\circ} \mathrm{C}\right)$ was subjected to western blot for detecting HIF-1 $\alpha$ (Abcam, ab85886) or HIF-2 $\alpha$ (Abcam, ab199). $75 \mu \mathrm{g}$ total protein from tissue homogenate was loaded into each well of 10\% SDS-PAGE gel. Both anti- HIF-1 $\alpha$ (Abcam, ab85886) and anti- HIF-2 $\alpha$ (Abcam, ab199) were used with a dilution rate of $1 / 1000$.

\section{Statistical analyses}

SPSS11.0 software was used for $t$ test or $t^{\prime}$ test (for unequal variances), and Pearson correlation analysis of the data.

\section{Results}

\section{The pregnancy-dependent expression of PHD in normal placenta}

We detected the expression of PHD-1 3 in the late pregnancy group by in situ hybridization. PHD-1, 2 and 3 mRNA were mainly present in placental trophoblast cells with cytoplasm location, especially in syncytiotrophoblast and trophoblastic cells (Figure 1). Villous stroma cells, probably endothelial cells or macrophages, also exhibited PHDs expression. Noteworthy, the highest expression of PHD-1 mRNA appeared in the cytoplasm of extravillous trophoblast (Figure 1B). The specifically expression of PHDs in placental trophoblast cells indicated that PHDs played an essential role in mediating the oxygen sensitivity during gestation and raised the possibility that PHDs involved in the pathology of preeclampsia.

Table I. Clinical Parameters Of Control And Preeclampsia.

\begin{tabular}{llll}
\hline & Control & Gestational hypertension & Preeclampsia group \\
\hline Case number & $\mathrm{n}=13$ & $\mathrm{n}=11$ & $\mathrm{n}=20$ \\
Mean maternal age(yr) & $29.54+2.40$ & $30.45+6.53$ & $27.77+4.29$ \\
G.A. at delivery $(\mathrm{wk})$ & $39.21+0.60$ & $39.71+1.40$ & $37.40+2.70$ \\
number of pregnancies & $2.08+1.38$ & $1.55+0.52$ & $1.73+0.94$ \\
Parity & $1+0$ & $657.73+93.28$ & $1.09+0.29$ \\
placental weight $(\mathrm{g})$ & $690.77+132.94$ & $3776.36+301.86$ & $598.45+163.97$ \\
Fetal weight(g) & $3560.00+333.39$ & Male: $5 ;$ Female: 6 & $3066.14+798.99$ \\
Fetal Sex & Male: 6 ; Female: 7 & $109.27+2.90$ & Male: $11 ;$ Female: 9 \\
MAP(mmHg) & $86.62+0.65$ & negative & $123.63+15.22$ \\
Proteinuria $(\mathrm{mg} / \mathrm{dl})$ & Negative & $151.14+127.35$ & \\
\hline
\end{tabular}

Control group is the late pregnant group. G.A.: Gestational Age. MAP: mean arterial pressure. 

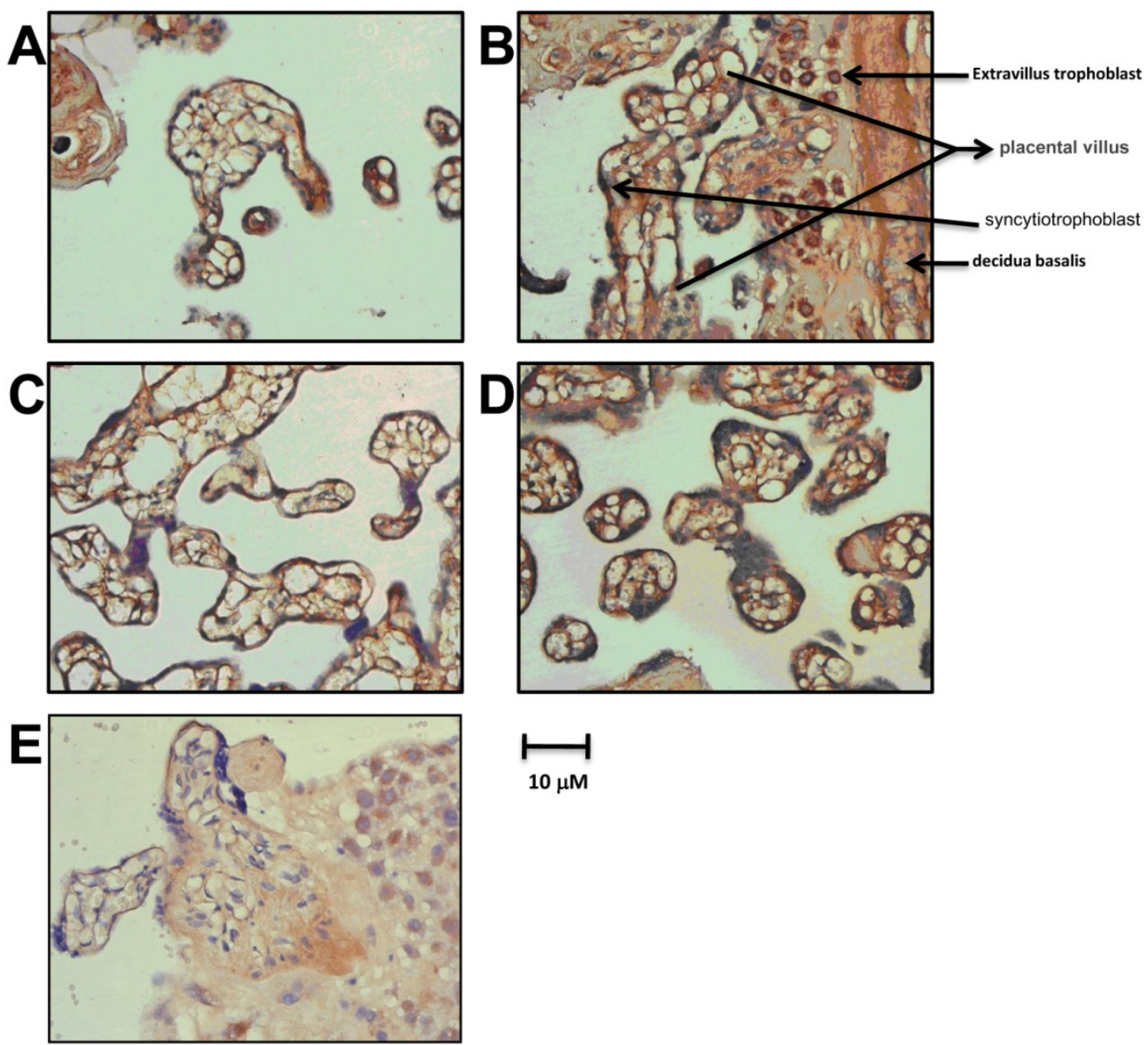

Figure I. The expression of PHDs mRNA in placenta of late pregnancy group. PHD-I mRNA expression in placenta from late pregnancy detected by in situ hybridization. B. The specific stain of PHD -I mRNA by in situ hybridization in extravillus trophoblast from late pregnancy group. C. PHD-2 mRNA expression in placenta from late pregnancy by in situ hybridization. D. PHD-3 mRNA expression in placenta from late pregnancy by in situ hybridization. $\mathrm{E}$. Blank Control with non-specific probe.

Then, we investigated the expression level of PHDs in the different stages of normal gestation. As the pregnancy progresses from early to middle to late pregnancy state, PHD-1 and -2 displayed gradually increasing expression level (Figure 2). In addition, PHD-1 expression levels showed a significant positive correlation with the progress of pregnancy $(R=0.616$, $\mathrm{P}<0.001)$. The expression of PHD-3 in middle pregnancy group was significantly higher than that in early pregnancy group (Figure 2). However, there was no difference in PHD-3 between middle and late pregnancy groups (Figure 2). The correlation analysis showed that the expression levels of the PHD-1 and PHD-2 displayed a significant positive correlation $(R=0.607, P<0.001)$, indicating that they may functionally overlap in the process of pregnancy. We proposed that the increasing expression of PHD-1 and -2 implied the enhanced oxygen sensitivity of trophoblastic cells. The diverse expression pattern of PHD-3 suggested that it may play a different role from that of PHD-1 or PHD-2 during gestation.

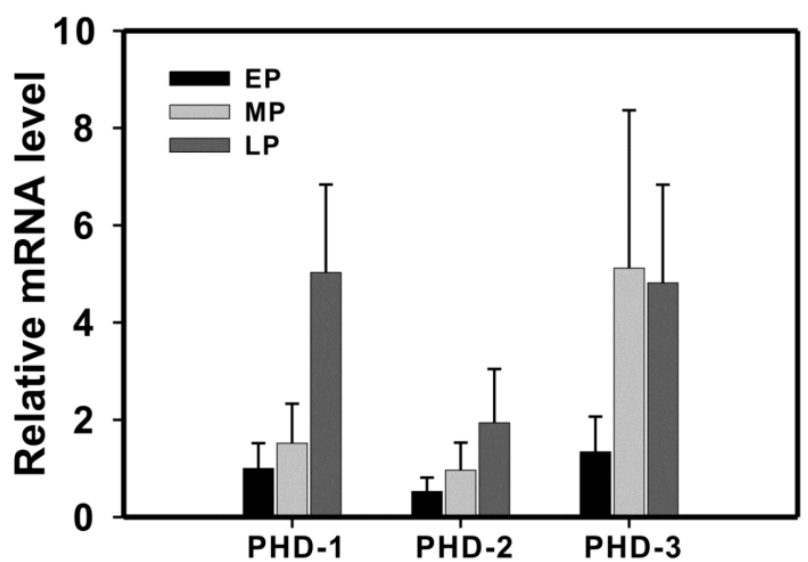

Figure 2: The expression of PHD-I, -2 and -3 mRNA in normal pregnancy. PHD-I, -2 and -3 mRNA expressions in early-, middle- and late-pregnancy groups were determined by real-time PCR analysis. All the mRNAs expression levels after rectified with that for GAPDH were normalized with that of PHD-I in early pregnancy group. Abbreviation: EP, early pregnancy group; MP, middle pregnancy group; LP, late pregnancy group. 


\section{The expression of PHDs in preeclampsia and gestational hypertension groups}

The level of PHD-1mRNA in preeclampsia group was significantly lower than that of control group $(\mathrm{P}<0.05)$ (Figure 3). Although PHD-1 of hypertension groups was also lower than control group, there was no statistically significant difference between them (Figure 3) $(\mathrm{P}<0.05)$. We also compared the expression level of PHD-2 and-3 between preeclampsia and control groups. However, they both showed no significant difference. There results showed that preeclampsia could significantly lead to PHD-1 low expression in placenta, while having minor effect on PHD-2 and -3. Among the clinical manifestations, placental weight was the only one having significant positive correlation with PHD- 1 mRNA ( $R=0.457$, $\mathrm{P}<0.05$ ) (Table 2). This meant that the decreased of PHD-1 mRNA expression would resulted in lighter placenta.

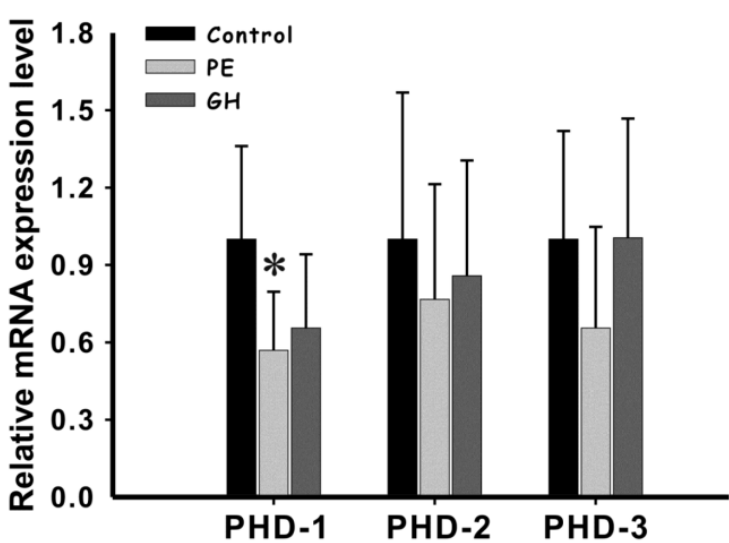

Figure 3: The expression of PHD-I and -2 and -3 mRNA in placenta from preeclampsia group and gestational hypertension group and control group. PHD- I, -2 and -3 mRNA expressions in control group, preeclampsia group and gestational hypertension group were determined by real-time PCR analysis (values are mean $\pm \mathrm{SEM},{ }^{*} \mathrm{p}<0.05$ ). All the mRNAs expression levels after rectified with that for GAPDH were normalized with that in control group. Abbreviation: $\mathrm{PE}$, preeclampsia group; $\mathrm{GH}$, gestational hypertension group.
Table 2: The correlation analysis between PHD-I mRNA and HIF-2 $\alpha$ protein in placenta from PE group and clinical manifestation.

\begin{tabular}{lllll}
\hline Clinical manifestation & \multicolumn{2}{c}{ PHD-1 } & \multicolumn{2}{c}{ HIF-2 $\alpha$} \\
\cline { 2 - 5 } & Value & $\mathrm{P}$ & Value & $\mathrm{P}$ \\
\hline 1MAP(mmHg) & -0.060 & 0.801 & -0.107 & 0.741 \\
$\mathrm{UPC}(\mathrm{mg} / \mathrm{dl})$ & 0.297 & 0.203 & -0.263 & 0.408 \\
WON(g) & -0.001 & 0.996 & -0.425 & 0.169 \\
PW(g) & $0.457^{*}$ & 0.043 & -0.143 & 0.658 \\
OT (day) & -0.075 & 0.752 & $-0.730^{* *}$ & 0.007 \\
AFT(limpidity, degree & 0.367 & 0.111 & 0.336 & 0.285 \\
1 3) & & & & \\
NAS(1 10) & 0.057 & 0.812 & -0.478 & 0.116 \\
FRG(Yes or No) & -0.097 & 0.683 & 0.358 & 0.253 \\
premature(Yes or No) & -0.228 & 0.334 & $0.591^{*}$ & 0.043 \\
ES(Yes or No) & -0.041 & 0.863 & 0.389 & 0.212 \\
\hline
\end{tabular}

The correlation analysis was performed with SPSS11.0 software. Abbreviation: MAP, mean arterial pressure; UPC, urinary protein concentration; WON, Weight of Newborns; PW, placenta weights; OT, Onset time; AFT, amniotic fluid turbidity; NAS, Neonatal apgar score; FRG, Fetal Growth Restriction; ES, Eye spasms. ${ }^{*} \mathrm{P}<0.01,{ }^{*} \mathrm{P}<0.05$.

\section{The protein expression level of HIF-I $\alpha$ and HIF- $2 \alpha$ in preeclampsia placenta}

As PHD directly involved in the degradation of HIF, we then investigated how the low level of PHD-1 in preeclampsia placenta affected the protein level of HIFs. The expression of both HIF- $1 \alpha$ and HIF- $2 \alpha$ in preeclampsia placenta was detected by immunohistochemistry. Both of them were predominately expressed in syncytiotrophoblast leaf cells and trophoblastic villus cells (Figure 4). HIF-2 $\alpha$ of preeclampsia group was significantly higher than that of control group $(\mathrm{P}<0.001)$ (Figure 5). However, HIF-1 $\alpha$ protein level showed no significant difference between these two groups (Figure 5). We also analyzed the correlation between HIF- $2 \alpha$ and clinical manifestations. HIF-2 $\alpha$ was correlated with both the onset time $(\mathrm{P}<0.01)$ and preterm birth $(\mathrm{P}<0.05)$. The relationship between preeclampsia onset time and the HIF-2a level was significantly negative correlation.

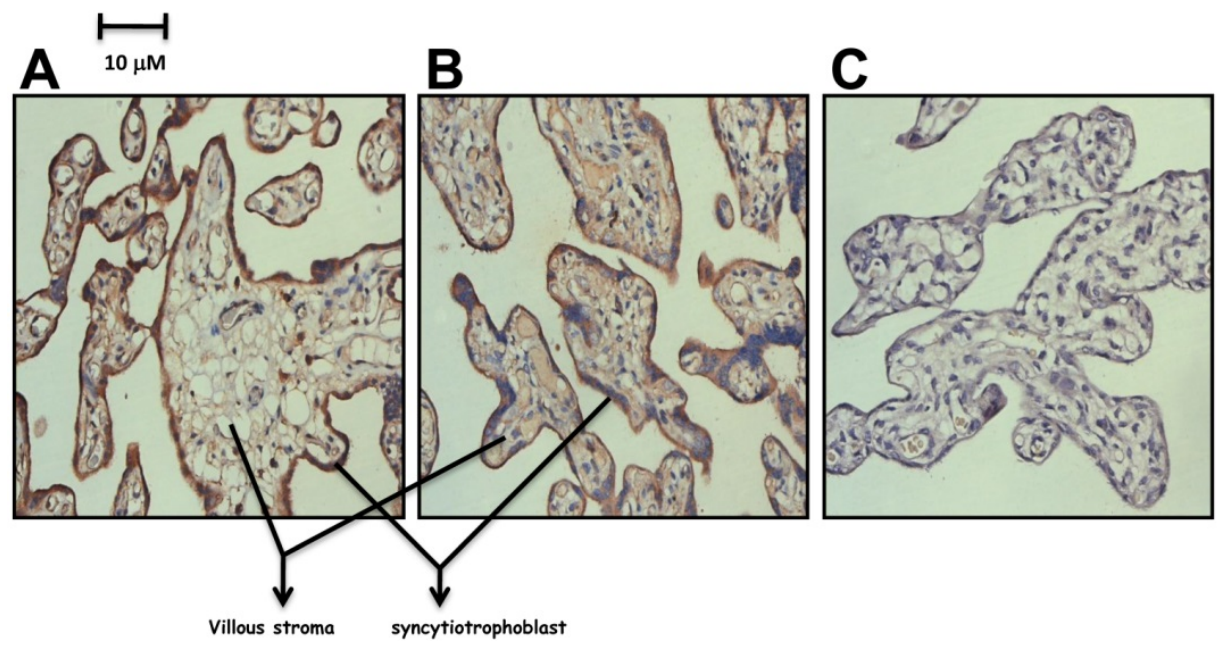

Figure 4: The expression of HIF-I $\alpha$ and HIF-2 $\alpha$ in preeclampsia placenta group. A. Detection of HIF-I $\alpha$ in preeclampsia placenta by Immunohistochemistry. B. Detection of HIF-2 $\alpha$ in preeclampsia placenta by Immunohistochemistry. C. Control with Rabbit lgG. 


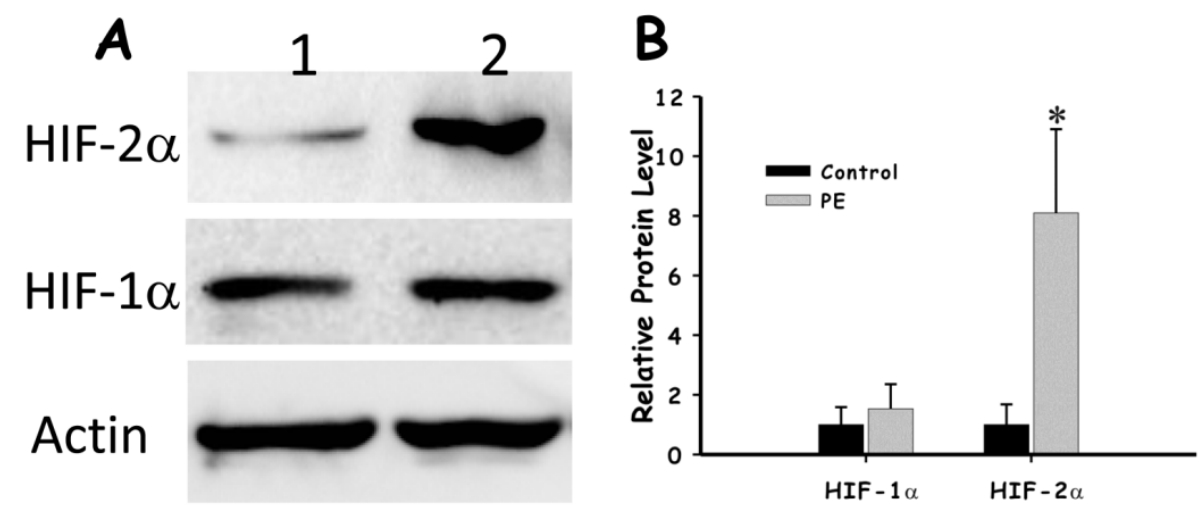

Figure 5: The comparison of HIF-I $\alpha$ and HIF-2 $\alpha$ in preeclampsia placenta group and control group. A. The expression of HIF-I $\alpha$ and HIF-2 $\alpha$ in preeclampsia placenta group and control group with Actin as control by western blot. B. Quantification of the expression of HIF-I $\alpha$ and HIF-2 $\alpha$ in preeclampsia placenta group and control group.

\section{Discussion}

Both physiological hypoxia stage in the normal pregnancy (before 12 weeks of pregnancy) and preeclampsia could induce the high expression of HIF- $\alpha$ in trophoblastic cells $[12,13]$. HIF- $\alpha$ played an important role in early placenta development [19]. The high expression of HIF- $\alpha$, which was an indicator of the over-activation hypoxia response, is thought to be a major cause of preeclampsia pathogenesis [11]. To elucidate the pathology of preeclampsia, it would be necessary to investigate the underlining mechanism which regulates the hypoxia response pathway mediated through HIF- $\alpha$ in placental trophoblastic cells.

PHD hydroxylates some specific prolines in the alpha subunit of the hypoxia-inducible transcription factor (HIF) and controls its stability in an oxygen-dependent manner. In normoxia two prolines in the oxygen-dependent degradation domain of the HIFa subunit become hydroxylated by the PHD. The 4-hydroxyproline residues of HIFa facilitate binding of HIFa to the von Hippel-Lindau E3 ubiquitin ligase complex leading to subsequent ubiquitination and instant proteasomal degradation in normoxia. The PHD reaction is inhibited in hypoxia, HIFa evades degradation and forms a functional dimer with HIF $\beta$ activating the HIF target genes. Therefore, PHD functions as effective oxygen sensor.

We present work firstly study the PHDs mRNA expression pattern during normal gestation. PHDs were mainly expressed in syncytiotrophoblast leaf cells and trophoblast cells with PHD-1 mRNA specifically highly expressing in extravillous trophoblast cells (Figure 1), suggesting that PHDs was closely related to trophoblastic invasion activity. As pregnancy processes, both the expression levels of both PHD-1 and PHD-2 mRNAs increase synchronously. The enhancement of PHD-1 mRNA is significant, while PHD-2 was not. The expression of PHD-3 reaches to the highest in middle pregnant group and do not increase anymore in late pregnant group. The different expression patterns of PHDs indicated that PHD-1 and PHD-2 cooperate to function in the growth and development of trophoblast cells. Previous work has revealed that PHD-1 and -2 are more efficient in hydroxylating Pro564 of HIF- $\alpha$ than PHD-3, and that only PHD-3 could not hydroxylate Pro402 [20]. As the pregnancy progresses, the trophoblast cells gradually increase the oxygen sensitivity. These results indicated that the oxygen sensitivity of trophoblastic cell was regulated by PHD during normal pregnancy process. In the early state of pregnancy, low oxygen-sensitive of trophoblastic cells leads to hypoxia Under hypoxic condition, the invasion activity of trophoblastic cell was significantly enhanced, which in turn is conducive to placental vascular remodeling process [21]. With the progress of pregnant progress as well as placental vascular remodeling, trophoblast cells gradually increase the oxygen sensitivity and switch to the high-oxygen state. Under that condition, invasion activity and the translation factor involving in hypoxia response were inhibited.

Through the investigation on the PHDs expression in preeclampsia, we find that PHD-1 mRNA in preeclampsia patients was significantly lower than that of normal pregnant women, implying that the declined oxygen sensitivity of preeclampsia placenta. However, pregnancy-induced hypertension patients exhibit no significant difference in PHD-1 level. These results show that the oxygen sensitivity of trophoblast cells in preeclampsia placenta is different from normal gestation. Preeclampsia patients still remain high oxygen sensitivity in the late state of pregnancy. As a consequence, HIF-2 $\alpha$ remains high expression level even under the adequate oxygen due to the declined PHD. Our work also show that HIF-1 $\alpha$ did not show 
abnormal expression in preeclampsia placenta, suggesting it does not involves in the pathological process. PHD-1 was correlated with placental weight but not the onset time and preterm birth. The decreased PHD-1 would lead to the abnormal accumulation of $\mathrm{HIF}$, which representatives a lower $\mathrm{O}_{2}$ tension. In this situation, hypoxia response would be continuously activated, which in turn altered the metabolism of placenta cell. We proposed that the altered metabolism pattern disturb the growth of placenta and led to lighter placenta.

HIF-2 $\alpha$ was correlated with both the onset time $(\mathrm{P}<0.01)$ and preterm birth $(\mathrm{P}<0.05)$. High level of HIF- $\alpha$ would activate its target hypoxia-related genes expression, including vascular endothelial growth factor (VEGF), erythropoietin, leptin and so on. These proteins would trigger systemic vascular endothelial cell dysfunction, leading to a series of clinical manifestations of preeclampsia. Based on the previous reports and our present work, we propose a possible model for the pathology of preeclampsia. Certain immune or genetic factors lead to functional deficiency of trophoblast cells, which in turn subsequently restricts the capability of trophoblastic invasion and placental vascular recast. In addition, the trophoblastic oxygen sensitivity was also inhibited in the late state of pregnancy, which was indicated by the low expression of PHD-1. As a consequence, HIF- $\alpha$ is pathologically accumulated, which finally leads to the onset of preeclampsia (Endothelial cell toxicity symptoms of trophoblastic hypoxia). The present work highlights that PHD and HIF could be the potential targets for preeclampsia therapy. To ultimately understand the preeclampsia pathogenesis, the functions of different proline hydroxylase family members on HIF regulation and placenta development as well as pathology of preeclampsia was worthy of further investigation.

\section{Supplementary Material}

Table S1, Table S2 and Fig.S1.

http://www.ijbs.com/v10p0278s1.pdf

\section{Acknowledgement}

This study was supported by Shanghai Education Commission Key Disciplines Foundation.

\section{Conflict of Interest}

The authors declare no conflict of interest.

\section{References}

1. Ghulmiyyah L, Sibai B. Maternal mortality from preeclampsia/eclampsia. Semin Perinatol 2012; 36: 56-59.

2. Moodley J. Maternal deaths associated with hypertensive disorders of pregnancy: a population-based study. Hypertens Pregnancy 2004; 23: 247-256.

3. World Health Organization. World health report 2005: make every mother and child count. Geneva: WHO. 2005.

4. Liang J, Li WM, Wang YP, et al. Analysis of national maternal mortality trend from 1996 to 2000. Chinese J Obstetrics Gynecol 2003; 38: 471-473

5. Shah DM. Preeclampsia: new insights. Curr Opin Nephrol Hypertens. 2007; 6: 213-220.

6. Bruick RK, McKnight SL. Transcription. Oxygen sensing gets a second wind. Science 2000; 295:807-808.

7. Shay JE, Celeste Simon M. Hypoxia-inducible factors: crosstalk between inflammation and metabolism. Semin Cell Dev Biol. 2012; 23: 389-394.

8. Patel J, Landers K, Mortimer RH, Richard K. Regulation of hypoxia inducible factors (HIF) in hypoxia and normoxia during placental development. Placenta. 2010; 31: 951-957.

9. Rajakumar A, Whitelock KA, Weissfeld LA, Daftary AR, Markovic N, Conrad $\mathrm{KP}$. Selective overexpression of the hypoxia-inducible transcription factor, HIF-2alpha, in placentas from women with preeclampsia. Biol Reprod. 2001; 64: 499-506.

10. Tal R. The role of hypoxia and hypoxia-inducible factor-1Alpha in preeclampsia pathogenesis. Biol Reprod. 2012; 87: 134

11. Caniggia I, Winter JL. Adriana and Luisa Castellucci Award lecture 2001. Hypoxia inducible inducible factor-1: oxygen regulation of trophoblast differentiation in normal and pre-eclamptic pregnancies--a review. Placenta, 2002; (Suppl A): S47-57.

12. Rajakumar A, Brandon HM, Daftary A, Ness R, Conrad KP. Evidence for the functional activity of hypoxia-inducible transcription factors overexpressed in preeclamptic placentae. Placenta. 2004; 25: 763-769

13. Rajakumar A, Conrad KP. Expression, ontogeny, and regulation of hypoxia-inducible transcription factors in the human placenta. Biol Reprod. 2000; 63: 559-569.

14. Ietta F, Wu Y, Winter J, Xu J, Wang J, Post M, Caniggia I. Dynamic HIF1A regulation during human placental development. Biol Reprod. 2006; 75: 112-121.

15. Hirsilä M, Koivunen P, Günzler V, Kivirikko KI, Myllyharju J. Characterization of the human prolyl 4-hydroxylases that modify the hypoxia-inducible factor. J Biol Chem. 2003; 278: 30772-80.

16. Bruick RK, McKnight SL. A conserved family of prolyl-4-hydroxylases that modify HIF. Science. 2001; 294: 1337-40.

17. Ivan $\mathrm{M}$, Haberberger $\mathrm{T}$, Gervasi $\mathrm{DC}$, et al. Biochemical purification and pharmacological inhibition of a mammalian prolyl hydroxylase acting on hypoxia-inducible factor. Proc Natl Acad Sci U S A. 2002; 99: 13459-64

18. Nakayama K, Frew IJ, Hagensen M, et al. Siah2 regulates stability of prolyl-hydroxylases, controls HIF1alpha abundance, and modulates physiological responses to hypoxia. Cell. 2004; 117: 941-52.

19. Adelman DM, Gertsenstein M, Nagy A, Simon MC, Maltepe E. Placental cell fates are regulated in vivo by HIF-mediated hypoxia responses. Genes Dev. 2000; 14: 3191-3203.

20. Koivunen P, Hirsilä M, Kivirikko KI, Myllyharju J. The length of peptide substrates has a marked effect on hydroxylation by the hypoxia-inducible factor prolyl 4-hydroxylases. J Biol Chem. 2006; 281: 28712-28720.

21. Graham CH, Fitzpatrick TE, McCrae KR. Hypoxia stimulates urokinase receptor expression through a heme protein-dependent pathway. Blood 1998; 91: 3300-3307. 